\title{
The implication of cancer regression by the interactions with human anatomical axes - an active prevention for cancer
}

\author{
Ming $\mathrm{COu}^{1,2 *}$ Dennis Ou ${ }^{3}$ and Chung $\mathrm{CPang}{ }^{4}$ \\ ${ }^{1}$ Department of Obstetrics and Gynecology, Taipei City Hospital, Taipei City, Taiwan \\ ${ }^{2}$ Department of Obstetrics and Gynecology, Taipei Medical University, Taipei City, Taiwan \\ ${ }^{3}$ Department of Mechanical Engineering and Biomedical Engineering, Carnegie Mellon University, Pittsburgh, USA \\ ${ }^{4}$ Department of Occupational Therapy, National Taiwan University, Taipei City, Taiwan
}

\begin{abstract}
Ou MC decrescendo phenomenon treatment (OuDPt) reduces pain or inflammation in an area by placing the contralateral hand over the lesion. OuDPt has been shown to have an anticancer effect, which may be due to tissue function normalization. Four cases of cancers that were treated with OuDPt were reported in this study. In two patients who received only OuDPt for anticancer treatment, one patient with stage IIIB uterine endometrial cancer and another with stage IVA ovarian cancer, the cancers regressed but later resumed growth despite continuing OuDPt. Another case of stage II uterine endometrioid adenocarcinoma showed prominent tumour regression after 8 months of OuDPt. After the initial tumour regression, the patient seldom performed OuDPt and the tumour resumed growth. The other case, stage III pancreatic cancer, showed decreasing levels of CA199 after combining OuDPt with chemotherapy. The resumed growth of this advanced cancer despite OuDPt may be due to poor accessibility of the tumour or escaping phenomenon through an unknown mechanism. Nonetheless, OuDPt shows a cancer suppression effect and can be performed by patients, which suggests that this therapy may be availed as a method of cancer prevention. Further study is warranted.
\end{abstract}

\section{Introduction}

Ou MC decrescendo phenomenon treatment (OuDPt), which can be administered by a patient or a therapist, is performed by placing the contralateral hand along human body anatomical axes (HBAAs) over a diseased location to produce a zone with less pain or inflammation under the hand $[1,2]$. OuDPt appears to be efficacious in treating a wide variety of diseases and has been evaluated in terms of distance between hand and lesion, treatment duration and performance frequency. Prompt remission of joint pain, reduced oedema of soft tissue induced by trauma, reduced pain caused by infection, cessation of uterine bleeding or cancer regression in studies with OuDPt indicate a restoration of normal tissue function [3]. Restoration of normal tissue function may re-establish host defence systems, contributing to defence against microorganisms, inflammation, degenerative changes, and tumours. Normalising the function of tumour cells may make them conform to apoptotic regulation, growth suppression, and metastatic hindrance as normal cells would. In this study, we observed the effect of OuDPt on 4 cases of cancer.

\section{Case report}

\section{Data collection}

Although complementary therapy does not fall under medical technology or care by law in Taiwan [4], all patients provided consent for OuDPt and for the details of their cases, including radiographic images, to be published.

\section{Methods}

$\mathrm{Ou} \mathrm{MC}$ decrescendo phenomenon is produced by placing the contralateral hand over a diseased location to produce a zone under the hand with decreased pain or inflammation [5-7]. From 2011 to 2015,
58 patients received OuDPt, and there was no difference in efficacy between self-administered (46/58) and therapist-administered (12/58) OuDPt ( $p>0.5,2$-sided), although OuDPt administered by a therapist may be influenced by other factors. In the group who self-administered OuDPt, the contralateral hand rather than ipsilateral hand showed more effectiveness ( $p<0.001,2$-sided) [5].

OuDPt for neoplastic diseases is mainly performed by patients placing the contralateral hand over the site of the lesion themselves $[1,6]$. While performing OuDPt, the contralateral hand is placed over the lesion along the left-right, dorsoventral or vertical HBAAs (Table 1). Three dimensional (axes) OuDPt has shown to be more effective than 2 dimensional (axes) OuDPt [6].

OuDPt showed short term therapeutic benefits for neoplastic disease in 10 patients of mean age 56.3 years (standard deviation, 10.3) in our studies from 2013 to 2018, and the data of cases 2, 5, 9 and 10 were updated and reported in this study (Table 2) $[2,3,5,6]$.

\section{Results}

As reported in our previous study, the neoplasms of cases 2 and 5 , which were stage IIIB uterine endometrial cancer and stage IVA ovarian cancer, respectively, initially reduced in size after OuDPt via 2 dimensions (2D) but later resumed growth and then shrunk again

*Correspondence to: Ming Cheh Ou, Department of Obstetrics and Gynecology, Taipei City Hospital, Taipei City, Xi Ning South Road, Lane 71, No.7, Taiwan, Tel: 886-979-308190; Fax: 886-2-23881498; E-mail: mcou@ym.edu.tw

Key words: barrett's esophagus, cancer, chemoprevention, mortality, prevention

Received: March 29, 2019; Accepted: April 12, 2019; Published: April 15, 2019 
Table 1. Practical points for Ou MC decrescendo phenomenon treatment (OuDPt) ${ }^{\mathrm{a}}$.

1.The OuDPt is mainly performed by the patient by placing the contralateral hand directly on the affected area via the left-right, dorsoventral or vertical axes. Application along more axes has been shown to be more effective.

2.For lesions on or adjoining the midline of the body, OuDPt is applied first to one side of the lesion and then the other, using the contralateral hand.

3.The effects of OuDPt are related to the duration and frequency of administration and the distance between the hand and the lesion.

4.If OuDPt is not efficacious, measures such as getting the hand nearer to the lesion and increasing the duration or frequency of administration may be helpful. Longer duration may cause organ compression and pressing too forcefully may cause tumour bleeding or exfoliation.

5.Different positioning may be useful when performing OuDPt.

6. Severe emotional disturbance may possibly affect effectiveness.

àodified from Ref 1,6 .

Table 2. Short term and long-term effects of Ou MC decrescendo phenomenon treatment (OuDPt) for cancer

\begin{tabular}{|c|c|c|c|c|c|c|c|}
\hline Case & Cancer & Age/Sex & Stage & Concomitant treatment & Treatment duration & Short term effect & Long term effect \\
\hline 1 & Uterine leiomyosarcoma & $59 / \mathrm{F}$ & IB & Nil & 1 month & $\begin{array}{l}\text { Stopping uterine } \\
\text { bleeding }\end{array}$ & n.a. \\
\hline $2^{\mathrm{a}}$ & Endometrial cancer $^{\mathrm{a}}$ & $49 / \mathrm{F}$ & IIIB & Nil & 4 years & Regression & Progression \\
\hline 3 & Suspected pancreatic cancer & $51 / \mathrm{F}$ & IA & Nil & 2 years & Regression & No recurrence \\
\hline 4 & $\begin{array}{l}\text { Suspected skin metastasis of } \\
\text { CML }\end{array}$ & $39 / \mathrm{F}$ & Chronic phase & Chemotherapy & 4 weeks & Regression $^{\S}$ & n.a. \\
\hline $5^{\mathrm{a}}$ & Ovarian cancer & $56 / \mathrm{F}$ & IVA & Nil & 2 years & Regression & Progression \\
\hline 6 & Uterine endometrial cancer & $53 / \mathrm{F}$ & IA & Nil & 2 months & $\begin{array}{l}\text { Stopping uterine } \\
\text { bleeding }\end{array}$ & n.a. \\
\hline 7 & Breast cancer & $74 / \mathrm{F}$ & IIB & Nil & 3 weeks & Regression & n.a. \\
\hline 8 & Pancreatic cancer & $63 / \mathrm{M}$ & III & n.a. & 1 month & Regression & n.a. \\
\hline $9^{\mathrm{a}}$ & Uterine endometrial cancer ${ }^{\mathrm{a}}$ & $50 / \mathrm{F}$ & II & Nil & 8 months & Regression & Progression $^{\mathrm{b}}$ \\
\hline $10^{\mathrm{a}}$ & Pancreatic cancer & 69/M & III & Chemotherapy & 2 weeks & Regression $^{\S}$ & n.a. \\
\hline
\end{tabular}

${ }^{a}$ The data of cases 2, 5,9 and 10 were updated and reported in this study. ${ }^{\mathrm{b}} \mathrm{After}$ tumour regression, OuDPt was seldom performed, and an MRI scan 5 months later showed the tumour had resumed growth. n.a: not available. CML: chronic myelogenous leukaemia.

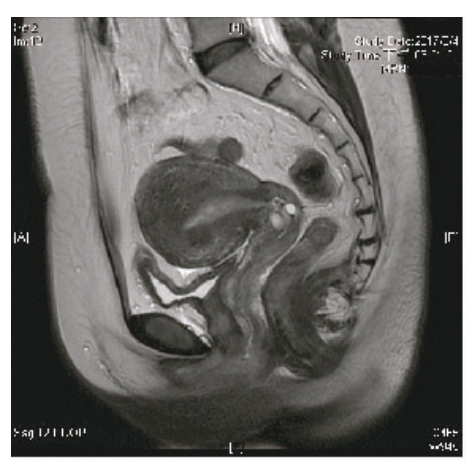

$2017 / 8 / 4(A)$

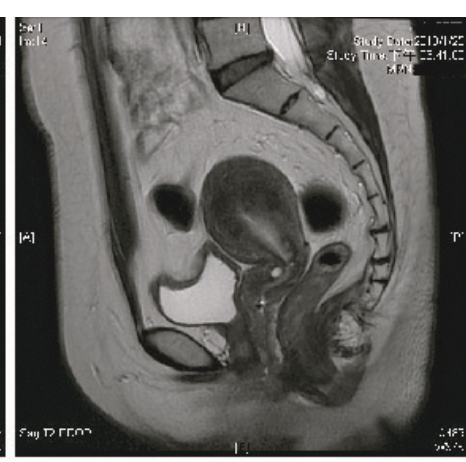

$2018 / 1 / 25(B)$

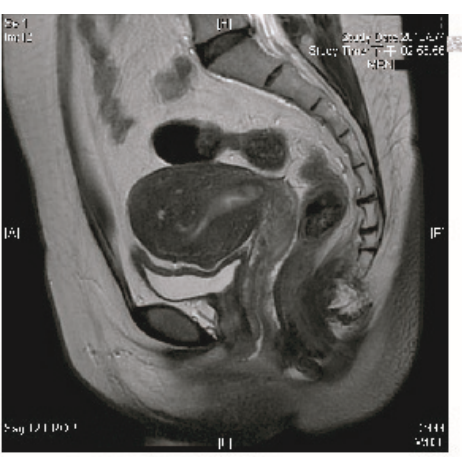

$2018 / 6 / 7 /(C)$

Figure 1. (A) MRI scan after Ou MC decrescendo phenomenon treatment (OuDPt) for stage II uterine endometrioid adenocarcinoma (grade 2) of case 9. (B) The tumour showed prominent regression after 6 months of OuDPt. (C) The tumour resumed growth when OuDPt was seldom performed.

following 3D OuDPt [6]. However, the advanced cancer of these two patients eventually resumed growth again despite the 3D OuDPt [8].

Case 9 was a stage II uterine endometrioid adenocarcinoma grade 2. This patient performed $2 \mathrm{D}$ OuDPt via left-right and dorsoventral axes 5 to 10 minutes on each site 2 to 3 times per day (Figure 1A). The endometrial tumour showed prominent regression after 8 months of treatment (Figure 1B). Thereafter, the patient seldom performed OuDPt, and an MRI scan 5 months later showed the tumour had resumed growth (Figure 1C).

Case 10 was a stage III pancreatic adenocarcinoma. The CA199 levels of this patient increased from $10,200 \mathrm{U} / \mathrm{ml}$ to $14,900 \mathrm{U} / \mathrm{ml}$ despite chemotherapy with weekly gemcitabine and daily TS-1 (a combination of tegafur, gimeracil and oteracil) for 3 weeks, and then decreased to $5,534 \mathrm{U} / \mathrm{ml}$ after adding $2 \mathrm{D}$ OuDPt [6] administered by the patient and a therapist for approximately 30 seconds to 1 minute on each site at least 2 to 3 times per day for 17 days.

\section{Discussion}

Most adult human cells and tissues have been found to be polarized, which enables their normal function. Pituitary homeobox 2 (PITX2), NODAL, and LEFTY-1 are important genes that control embryonic development along the left-right axis in mammals and have been found to be related to the development or suppression of various cancers, suggesting therapeutic relevance. For example, PITX2 protects renal cancer cell lines against doxorubicin toxicity, NODAL promotes breast cancer growth or progression, and LEFTY-1 expression is suppressed in induced pluripotent cancer cells [9-11]. A large number of signalling molecules that are active during the specification of embryonic leftright asymmetry are also active during tumour progression. It can thus be hypothesized that the occurrence of cancer is associated with signalling molecules related to embryonic left-right axis development.

Recent studies have shown that the signalling system of embryonic axes imparts polarization of individual cells in Drosophila [13]. 
Mammals also have multiple versions of each Drosophila polarity gene. Such polarity systems also play an important role in normal cell functions of all vertebrates, including humans, from the earliest stages of embryonic development to adult life. Thus, HBAAs may also impart polarity to individual cells just as embryonic axes do [3].

Disruption of polarity in cancer cells results in these cells losing their ability to behave normally in response to physiological cues and is frequently assumed to be a common feature of cancer progression. However, if cancer cell polarity can be normalised or reinforced, it may also normalise the function of these mutant cells. When the functions of tumour tissues are normalised, tumour cells may conform to apoptotic regulation, growth suppression, and metastasis suppression, processes which normal cells undergo. The normalisation of tumour tissue function may involve not only tumour cells but also the microenvironment in which the tumour cells are located. The tumour microenvironment has been regarded as a main factor in tumour development and metastasis. By normalizing the tumour microenvironment, tumour cells are subjected to an environment that suppresses metastasis, prevents uninhibited proliferation, minimizes angiogenesis, and eliminates abnormal cells via the normalised host immunological system. In our studies, OuDPt can result in prompt remission of joint pain, reduction of oedema of soft tissue caused by trauma, reduction in pain caused by infection, cessation of uterine bleeding or cancer regression, which indicates a restoration of normal tissue function $[1,2]$.

Our study has shown that OuDPt along the 3D human body polarity system composed of left-right, dorsoventral and vertical HBAAs suppressed neoplasm development more efficiently than OuDPt along the $2 \mathrm{D}$ polarity body system, providing evidence that the effect of OuDPt is associated with interaction of HBAAs [2,5]. 3D tissue organization has been reported to be a potential non-canonical tumour suppressor that prevents the manifestation of neoplastic features in mutant cells and, ultimately, suppresses tumour development and progression [13]. The interactions among multiple HBAAs with OuDPt also renders a $3 \mathrm{D}$ organization model that, similar to $3 \mathrm{D}$ tissue organization, is hypothesized to reinforce cell polarity of mutant cells and attenuate proliferation and invasion of the variable morphogenic mutant cells $[6,13]$. The tumour regresson by OuDPt may indicate that disruption of cell polarity is not only a by-product of cancer cells but also plays a causal role of cancer initiation and development.

Though OuDPt appears to be consistently effective for treating a wide variety of diseases, normalization of tissue function may not always be sufficient to resolve diseases. OuDPt is effective in treating pain induced by dysfunctional tissue such as that of degenerative joint disease or endometriosis but may not be sufficient to treat a disease unrelated to tissue dysfunction such as an infection [2,7]. OuDPt shows a suppressive effect on advanced cancer, but the effect may not extend to poorly accessible lesions. Though the appropriate duration, frequency, and method of OuDPt application for the treatment of different diseases requires further study, longer duration of OuDPt may cause organ compression and pressing too forcefully during OuDPt may possibly cause tumour bleeding or exfoliation, which can cause metastasis $[3,5,6]$.

OuDPt shows a suppressive effect on tumour growth, which may prevent cancer occurrence or even cure early stage cancers. Because OuDPt is easy to perform and can be done by patients themselves, OuDPt may be availed as a low-cost cancer prevention method. However, further study is warranted.

\section{Acknowledgement}

A part of this study was presented at the 17th Biennial Meeting of the International Gynecologic Cancer Society in Kyoto in 2018 [8]. The authors thank Ms. Yi Jen Ou for help with this manuscript.

\section{References}

1. Ou MC, Pang CC, Ou D, Su CH (2012) The implications of abdominal palpation with Ou MC manipulation for women with acute abdomen. Am J Emerg Med 30: 421-425. [Crossref]

2. Ou MC, Ou D, Pang CC (2017) The physiological reaction by interaction of human body anatomical axes results in tissue function normalization - a feature of human body axis sensation. Acta Physiol 221: 250.

3. Ou MC, Ou D, Pang CC (2016) Abstract 1436: The possible role of embryonic polarity axes for the normalization of tissue function induced by the interaction between human bilateral parts. Cancer Res 76: 1436.

4. Department of Health, Executive Yuan, Taiwan ROC (1993) Document No. 8207565. Department of Health, Executive Yuan, Taiwan ROC

5. Ou M, Ou D, Pang C (2015) The possible role of embryonic polarity axes for the normalization of tissue function induced by the interaction between human bilateral parts. Integr Cancer Sci Therap 3: 329-335.

6. Ou MC, Ou D, Pang CC (2017) Implication of tumor regression by a three-dimensional human body polarity system formed with human body anatomical axes. Taiwan $J$ Obstet Gynecol 56: 116-119. [Crossref]

7. Ou M, Ou D, Pang C (2018) The anti-inflammatory effect by interactions of human body anatomical axes for dysmenorrhea caused by endometriosis. J Assist Reprod Genet 35: 2097.

8. Ou M, Ou D, Chu Pang C (2018) The implication of tumor regression associated with tissue function normalization by human body anatomical axes interaction - A way for cancer prevention. Int J Gynecol Cancer 28: 492.

9. Lee WK, Chakraborty PK, Thevenod F (2013) Pituitary homeobox 2 (PITX2) protects renal cancer cell lines against doxorubicin toxicity by transcriptional activation of the multidrug transporter ABCB1. Int J Cancer 133: 556-567. [Crossref]

10. Quail DF, Zhang G, Walsh LA, et al. (2012) Embryonic morphogen nodal promotes breast cancer growth and progression. PLoS One 7: e48237. [Crossref]

11. Saito A, Ochiai H, Okada S, Miyata N, Azuma T (2013) Suppression of Lefty expression in induced pluripotent cancer cells. FASEB J 27: 2165-2174. [Crossref]

12. Gray RS, Roszko I, Solnica-Krezel L (2011) Planar cell polarity: Coordinating morphogenetic cell behaviors with embryonic polarity. Dev Cell 21: 120-133. [Crossref]

13. Lee M, Vasioukhin V (2008) Cell polarity and cancer--cell and tissue polarity as a noncanonical tumor suppressor. J Cell Sci 121: 1141-1150. [Crossref]

Copyright: (C2019 Ou MC. This is an open-access article distributed under the terms of the Creative Commons Attribution License, which permits unrestricted use, distribution, and reproduction in any medium, provided the original author and source are credited. 